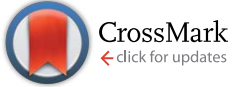

Cite this: RSC Adv., 2015, 5, 100790

Received 11th May 2015

Accepted 18th November 2015

DOI: $10.1039 / c 5 r a 08742 b$

www.rsc.org/advances

\section{Electron transport through electrically conductive nanofilaments in Rhodopseudomonas palustris strain RP2†}

\author{
Krishnaveni Venkidusamy, ${ }^{\text {ab }}$ Mallavarapu Megharaj, ${ }^{\text {abc }}$ Uwe Schröder, ${ }^{d}$ \\ Fouad Karouta, ${ }^{e}$ S. Venkata Mohan ${ }^{f}$ and Ravi Naidu ${ }^{\text {abc }}$
}

Electronic dialogue between proteins is expected to be a key component of charge transport at the microbe-mineral interface (MMI) and requires complex structures. Microbial nanofilaments are one such structure produced in energetically engineered environments. These nanostructures consist of natural protein electronic conduits which can target the microbe-mineral interface and facilitate charge transport over a distance. Nanofilaments are phylogenetically diverse inducible extracellular appendages, and have the potential to serve as organic electronic conductors. However, recent investigations on such microbial nanofilaments have been confined to a few bacterial genera such as Geobacter, Shewanella and Synechocystis. Here, we report the evidence for longitudinal electron transport through inducible nanofilaments produced by another genus, the metabolically versatile photosynthetic, iron(III) respiring bacterium Rhodopseudomonas palustris strain RP2, in photic, iron(III) oxide-rich environments. In contrast, chemosynthetic dark-grown anoxic cells are weak in their ability to reduce ferric-oxide and no longer produce extracellular structures. Independent evaluation techniques illustrate the induction of extracellular filaments and their electrical properties. Scanning probe and nanofabricated electrode measurements provide conclusive evidence for the occurrence of direct charge transfer along the length and radius of nanofilaments from strain RP2. These findings not only expand our knowledge of the range of bacteria known to produce nanofilaments but also provide further research opportunities in the field of bionanotechnology, sustainable remediation (bioelectrochemical remediation systems) in contaminated sites (petroleum hydrocarbons) and MMI process at photic environments.

\section{Introduction}

The interactions occurring at the microbial-mineral (MMI) interface have recently emerged as a field of study in electromicrobiology. This activity is fast becoming recognised as important in advanced technologies such as those used in bioelectrochemical remediation in challenging

${ }^{a}$ Centre for Environmental Risk Assessment and Remediation (CERAR), University of South Australia, Mawson Lakes, SA 5095, Australia. E-mail: megh.mallavarapu@ newcastle.edu.au

${ }^{b}$ CRC for Contamination Assessment and Remediation of the Environment (CRCCARE), Mawson Lakes, SA 5095, Australia

${ }^{c}$ Global Centre for Environmental Remediation, Faculty of Science and Information Technology, The University of Newcastle, Callaghan, NSW2308, Australia

${ }^{d}$ Institute of Environmental and Sustainable Chemistry, Technische, Universitat Braunschweig, Hagenring 30, 38106 Braunschweig, Germany

${ }^{e}$ Australian National Fabrication Facility, ACT Node, Australian National University, Canberra, Australia

${ }^{f}$ Bioengineering and Environmental Sciences (BEES), CSIR-Indian Institute of Chemical Technology (CSIR-IICT), Hyderabad 500 007, India

$\dagger$ Electronic supplementary information (ESI) available. See DOI: 10.1039/c5ra08742b environments. ${ }^{1-3}$ The microbial metabolism of solid minerals as final electron acceptors during energy conservation processes is known as the dissimilatory metal oxide reduction (DMR) pathway. ${ }^{\mathbf{1 , 4}}$ This pathway is an essential part in the remediation of organic contaminants, radioactive compounds and heavy metals such as uranium and chromium ${ }^{5,6}$ and it also assists in nutrient cycling ${ }^{\mathbf{1}}$ environmental sustainability and energy recovery processes. ${ }^{2}$ Dissimilatory metal oxide reducing bacteria (DMRB) are ubiquitous in nature ${ }^{7,8}$ and their identification ${ }^{9-11}$ has led to deeper insights into the molecular and physiological processes ${ }^{12,13}$ involved in electron transfer ${ }^{14}$ including how they interact with challengeable environments such as in presence of insoluble metal oxides, physical availability of electron acceptors. The insoluble nature of solid oxides requires peculiar structures to facilitate the transport of electrons from the bacterial inner membrane to these terminal acceptors. ${ }^{12,15,16}$

Recent investigations into extracellular electron transfer have established the importance of outer membrane cytochrome proteins ${ }^{12,16,17}$ electrically conductive extracellular appendages $^{\mathbf{1 0 , 1 1}}$ soluble mediators, ${ }^{18}$ secondary metabolites ${ }^{19}$ and chemotaxis ${ }^{20}$ in the metal oxide respiring or electrode respiring process. Of these, electrically conductive extracellular 


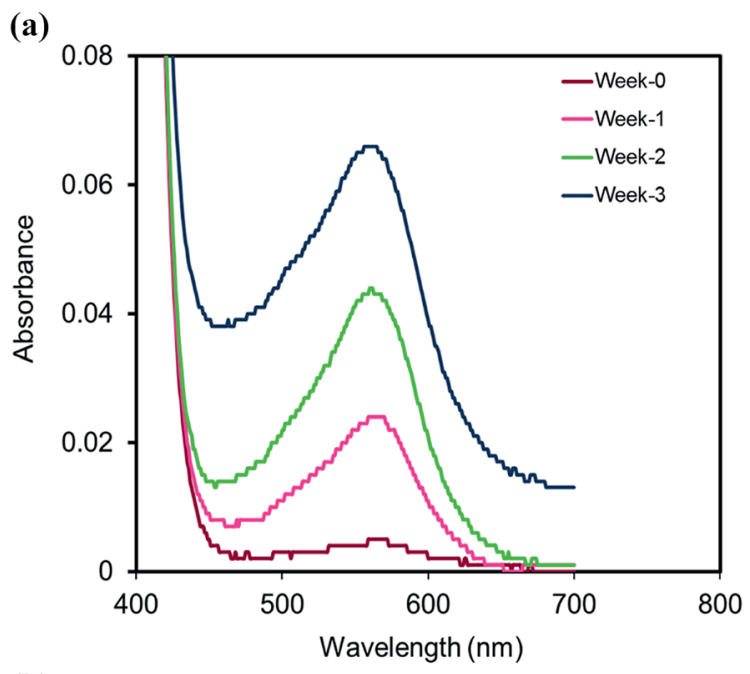

(b)

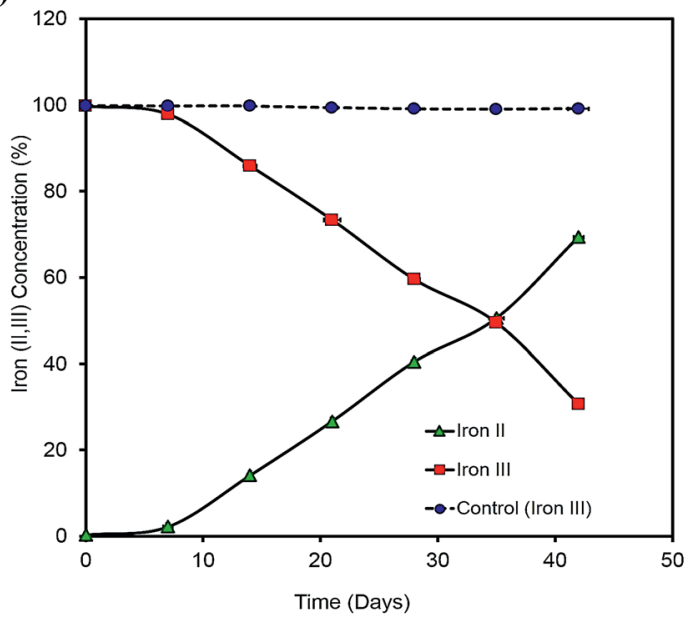

(c)

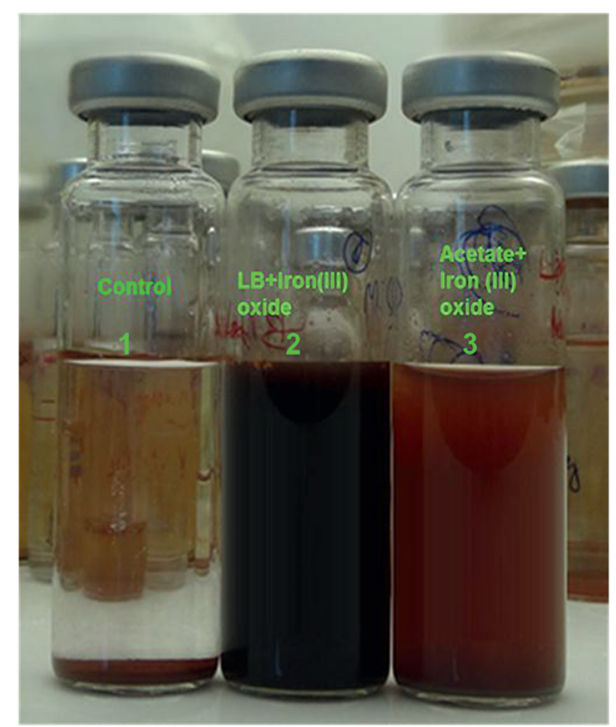

Fig. 1 (a) Dissimilatory iron(III) oxide reduction in anoxic photosynthetic incubated cultures at designated intervals (ferrozine assay). (b) Dissimilatory iron(III) oxide reduction in anoxic photosynthetic incubated RP2 cells at designated intervals (ferrozine assay, Fe(॥) formation, blue circle represents iron(III) reduction in chemosynthetic grown control cells; orange square represents iron(III) reduction in anoxic photosynthetic cells; green triangle shows iron(॥) formation in anoxic (induced) nanostructures consist of natural protein electronic conduits called bacterial nanofilaments which can target the microbe-mineral interface and facilitate charge transport over a distance during the MMI process. ${ }^{21}$ However, extensive studies have so far examined only a few bacterial nanofilaments, mainly from strains of the Geobacteraceae. ${ }^{22,23}$ Shewanellaceae, ${ }^{11,12}$ Merismopediaceae and in a syntrophy. ${ }^{24}$ Here we report the evidence for the induction of electrically active filaments from a metabolically versatile strain of Rhodopseudomonas palustris strain RP2 in energetically engineered environments.

\section{Results and discussion}

Rhodopseudomonas species are associated with a variety of environments such as euxinic lagoons, ${ }^{25}$ limnetic zones, ${ }^{26}$ marine sediments, sewage sludges and poorly drained soils. ${ }^{27}$ In our laboratory experiments, a strain of Rhodopseudomonas palustris, designated RP2 emerged as a dominant species isolated from the anodic biofilm of a previously enriched diesel-fed microbial fuel cell. Pure cultures of the strain RP2 contain double membrane bilayers, lamellar thylakoid membrane systems (ESI, Fig. S1(a)†), produce chains of magnetosomes (ESI, Fig. S1(b)†), cysts (ESI, Fig. S1(c) $\dagger$ ) and grow as long bacillus-shaped cells with asymmetric cell division(ESI, Fig. S1(d)†). Light grown anoxic cells are purple in colour and have exceptional growth flexibility based on environmental signals such as photoorganotrophic, photolithotrophic, dark fermentative and aerobic heterotrophic mechanisms. Salient properties of the strain RP2 were direct electrode respiration, dissimilatory metal oxide reduction, anaerobic nitrate reduction, free living diazotrophy and the ability to degrade $n$-alkane components of diesel range hydrocarbons in anoxic environments.

We cultured RP2 cells in LB medium under two different environmental conditions: anoxic photoheterotrophic and anoxic chemoheterotrophic with crystalline iron(III) oxide as a terminal electron acceptor to investigate the dissimilatory metal oxide reduction trait. Iron(III) oxide reduction was monitored by colour change (Fig. 1a) and hydroxylamine $\mathrm{Fe}(\mathrm{II})$ extraction assay (Fig. $1 \mathrm{~b}$ and c). Iron(III) oxide reduction (69.46\% $\pm 0.41 \%)$ was observed only in anoxic photoheterotrophic environments whereas chemosynthetic grown cells showed no reduction. During this process, peculiar extracellular filamentous structures were observed in the phototrophic growth conditions.

\subsection{Electron microscopy studies}

Scanning electron microscopy observation was used to determine that these extracellular structures were induced by physiologically relevant conditions. Priority was given to the culture

photosynthetic grown cells). (c) Visual indication of strain RP2 cells cultivated under anoxic phototrophic and chemotrophic with iron(III) oxide electron acceptor conditions. (1) Chemosynthetic sample with no iron reduction; ( 2 and 3 ) photosynthetic growth of strain RP2 cells with iron(III) oxide. 


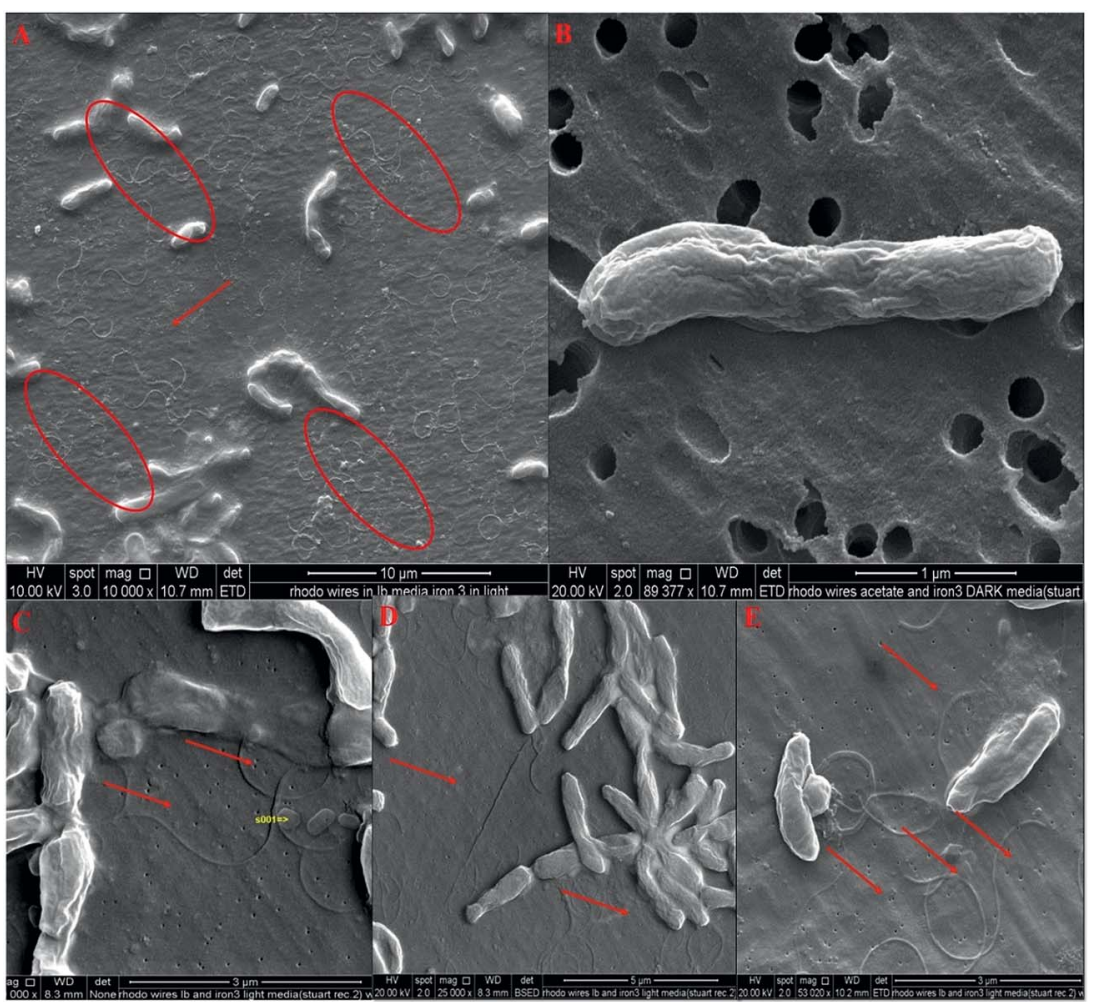

Fig. 2 Induction of extracellular filaments in anoxic photoheterotrophic cultures. (A) Expression of a number of filaments emanating from cells in photoheterotrophic, iron(III) oxide culture electron acceptor conditions - red circle indicates a high number of filaments induction under photoheterotrophic conditions. (B) Chemosynthetic anoxic culture conditions with no observed filaments. (C-E) The closer view of different sized nanofilaments induction under anoxic photoheterotrophic conditions indicated by red arrows.

conditions, and to using microscopic methods that avoided any artefacts resulting from polysaccharide layers as investigated earlier. ${ }^{10,11,24,28}$ During SEM examination, the largest numbers of extracellular filamentous structures were observed under phototrophically grown iron(III) oxide culture conditions (Fig. 2a-d). By contrast, cells grown in chemosynthetic environments were weak in their ability to reduce ferric oxide and no longer produced extracellular structures (Fig. 2e). The dimensions of these filaments are $>150 \mathrm{~nm}$ and they extend to several microns (Fig. 2b) with branches and bundles also occurring. Similar structures were perceived in transmission electron micrographs (ESI Fig. S2 $\dagger$ ) with regular shaped chains of magnetite as magnetosomes (ESI Fig. S1(b) †) running along the longitudinal axis of cells. These results indicated that the extracellular appendages were clearly inducible under relevant physiological conditions involving solid electron acceptor reduction as reported in earlier studies of Geobacter ${ }^{10}$ and Shewanella. ${ }^{11,24}$

\subsection{Scanning probe imaging studies}

To further investigate the filaments' electrical conductance properties, they were characterized by employing a number of independent methods including scanning probe imaging with both current sensing atomic force microscopy (CSAFM) and scanning tunnelling microscopy (STM). In order to verify the evidence of longitudinal electron transport of RP2 nanofilaments, we used nanofabricated electrodes combined with a dual beam focused ion beam technique (FIB) with SEM capability.

2.2.1 Atomic force microscopy (AFM). Phototrophically grown anoxic cells were then applied to a newly cleaved highly crystallographic oriented graphite surface (HOPG) and prepared as described earlier. Atomic force microscopic analysis of airdried HOPG samples showed that the filaments' height starts from $10 \mathrm{~nm}$ above the graphite surface, as presented in ESI Fig. S3.广

2.2.2 Current sensing AFM (CSAFM). For CSAFM, conductive mapping was carried out by scanning across the bacterial filament and this showed a clear peak current with a positive current response corresponding to the applied positive voltage and vice versa (Fig. $3 \mathrm{a}$ and $\mathrm{b}$ ). Further imaging of the samples at different locations showed a number of conductive extracellular appendages around the cells. Topographical imaging revealed that the thick bundles covered by a sheath of soft materials, were possibly either from suspended material or extracellular polymers.

2.2.3 Scanning tunnelling microscopy (STM). STM images showed that the height of extracellular appendages' was close to the real height with a current ranging from $20 \mathrm{nA}$ and they have a bundled structure along with their entire length (Fig. 3c and d). The diameter of the filament starts from $150 \mathrm{~nm}$ and $20-$ $40 \mathrm{~nm}$ in height. Height analysis of the insulating materials around the nanofilaments or on the surface of HOPG resulted in a zero value (ESI Fig. S4†). These measurements suggest that 

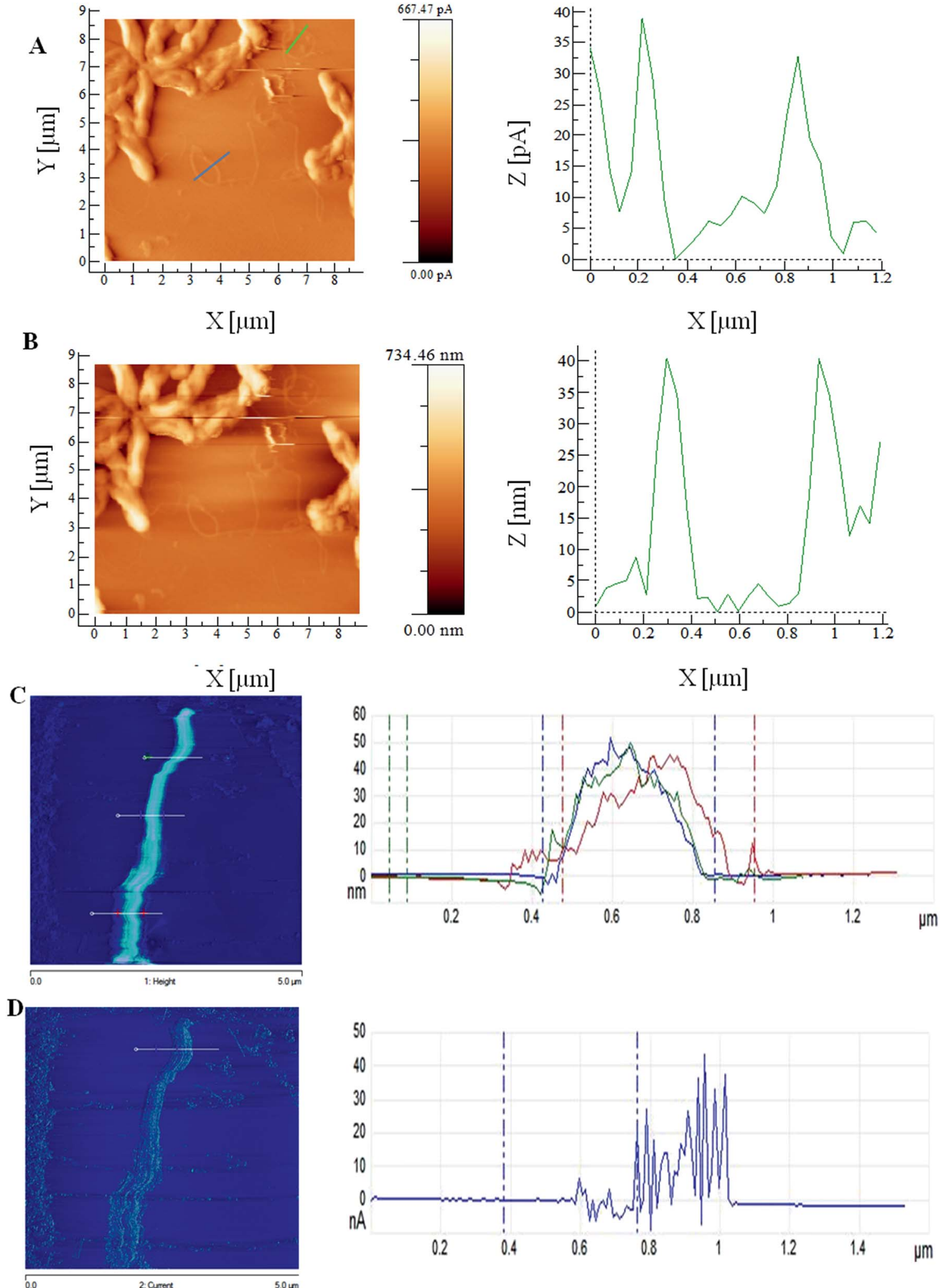

Fig. 3 Scanning probe microscopy techniques (A and B), current sensing AFM imaging shows height and current profile of nanofilaments from Rhodopseudomonas palustris strain RP2. (A) Relationship between current and distance. (B) Relationship between current and height profile. (C and D) Height and current profile of bacterial nanofilaments obtained from STM. (C) Topography and height of a nanofilament profile from STM, (D) STM topography and current profile confirm the induced nanofilaments are electrically conductive. 
A

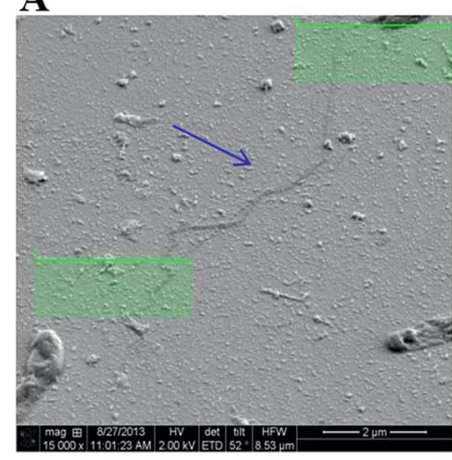

\section{C}

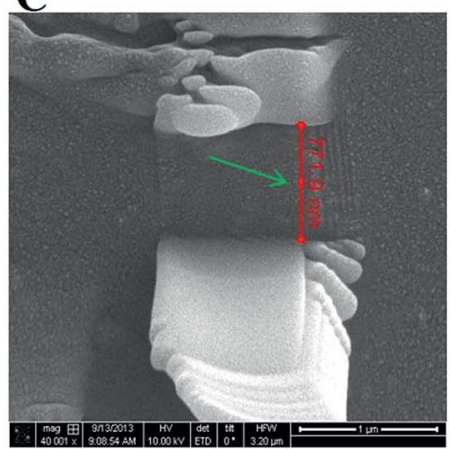

D

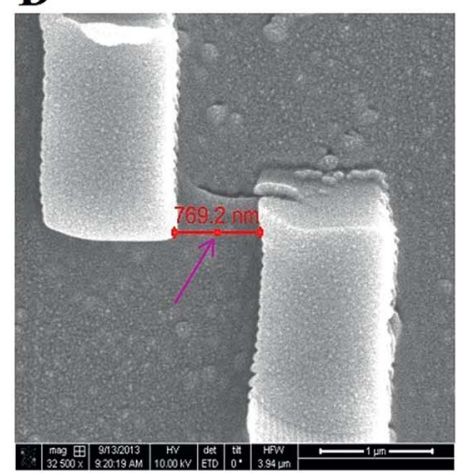

B
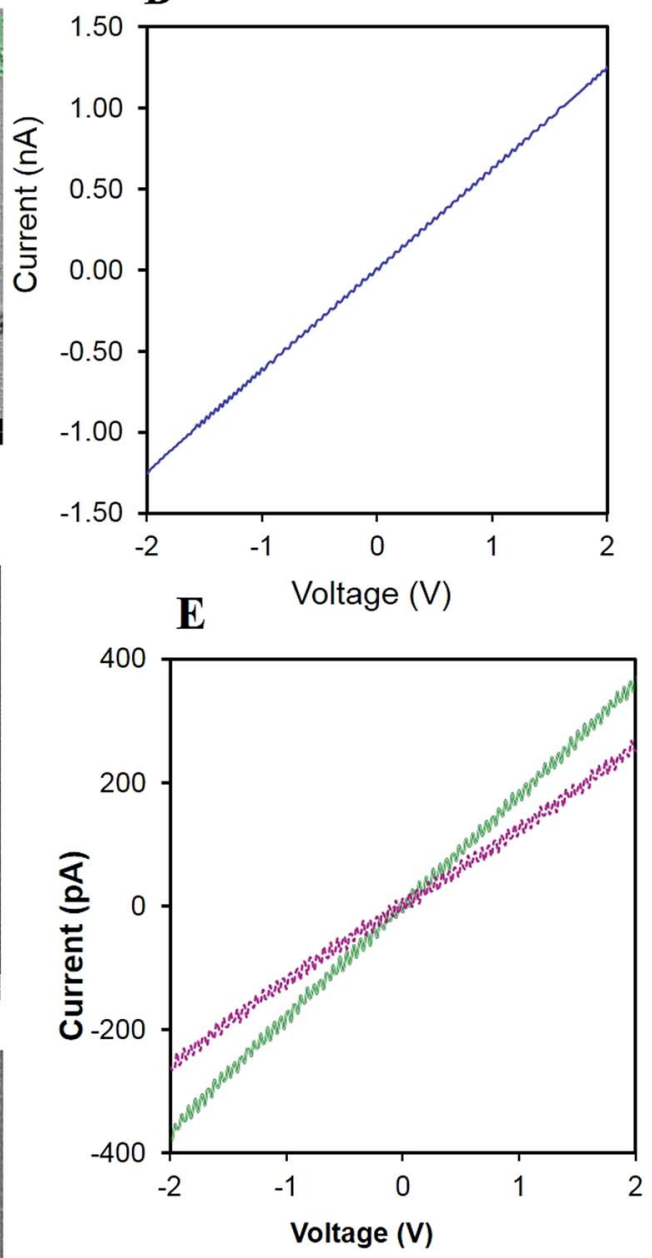

Fig. 4 Electrical measurements of bacterial nanofilaments using nanofabricated devices (A) FIB-SEM image showing a single nanofilament with the two edges contacted through Pt deposition in the FIB (B) shows current-voltage measurement of the same nanofilament (C and D) shows two other contacted and shorter nanofilaments (E) shows their current-voltage characteristics.

RP2 filaments are electrically conductive and are hence can be designated as nanofilaments.

\subsection{Electrical measurements using nanofabrication electrodes by dual beam focused ion beam method}

A nanofabricated electrode technique was applied by using a combination of lift-off technique, optical lithography and FIB technique to ensure electrical contact with the nanofilaments using Pt deposition. ${ }^{29}$ This technique has been demonstrated by previous researchers ${ }^{\mathbf{1 1}, 28}$ who evaluated the electrical conductance of semiconductor nanowires ${ }^{30}$ and biological nanowires in the Shewanella and the dental microbiome. ${ }^{11,28}$ Chemically fixed uncoated nanofilament samples were placed on the glass substrate with pre-patterned $\mathrm{Au}$ microelectrodes. The dual beam focused ion beam SEM imaging was used to identify the positions of nanowires located either between two adjacent contact pads or touching one contact pad. ESI Fig. S5† shows a FIB-SEM micrograph of the overall layout with all contacted nanofilaments and the reference strip with the contact pads. $I / V$ measurements show a strong linear relationship following the Pt deposition with an applied voltage from $-2 \mathrm{~V}$ to $+2 \mathrm{~V}$. The closed circuit reference indicated a resistance of $1.8 \mathrm{G} \Omega$ from a current of $550 \mathrm{pA}$ at $1 \mathrm{~V}$. Fig. 4a illustrates the contacting procedure of a single nanofilament by Pt electrode originating from strain RP2 grown under physiologically relevant conditions. The measured length of nanofilament 1 was $5.6 \mu \mathrm{m}$ with a calculated resistance of $2.96 \mathrm{G} \Omega(1 \mathrm{~V})$. Fig. $4 \mathrm{~b}$ illustrates the linear relationship from two other bacterial nanofilaments emanating from the same sample with a resistance of $3.22 \mathrm{G} \Omega$, 
$1.52 \mathrm{G} \Omega$ and resistivity of $290 \Omega \mathrm{m}^{-1}, 139 \Omega \mathrm{m}^{-1}$ respectively. The ohmic relationship between $I$ and $V$ confirmed the conductive nature of the nanofilaments and the response was similar to that seen in Shewanella ${ }^{11}$ and oral microbiome ${ }^{28}$ studies.

The bacterial nanofilaments were tens of nanometres to tens of micrometres longer than the bacterial cells. These findings suggest that the bacterial nanofilaments are distinctive extracellular appendages that function like a conductor and are consistent with earlier reports for other organisms. ${ }^{22,31}$ These conductive filaments are not only exclusive to environmental isolates from dissimilatory bacteria, but are also produced in biofilms such as oral microbiome. ${ }^{28}$ Such nanofilaments make electrical communication between cells possible. ${ }^{21}$ These nanofilaments also differ within the dissimilatory metal reducers. The occurrence of thin single-strand nanofilaments has been observed in Geobacter sulfurreducens ${ }^{\mathbf{1 0}}$ whereas Shewanella oneidensis ${ }^{\mathbf{2 4}}$ produced thick cable-like conductive wires to obtain access to the insoluble electron acceptors. In our study, we have observed both single-strand and bundled structures with a conductive trait. This may be due to variations in the pilin domain proteins between each group of organisms. ${ }^{10,24,32}$ Type IV pilin proteins of Shewanella oneidensis are longer than Geobacter pilin proteins such as fimbrium. ${ }^{\mathbf{1 0}}$ Similarly, a distant phylogenetic relationship exists between the gene $\operatorname{xxp} \mathrm{G}$ in Geobacter and the gene involved in type IV bacterial pilin secretion. ${ }^{\mathbf{1 0 , 2 4}}$

\subsection{Analysis of key proteins involved in extracellular electron transfer mechanisms}

The outer membrane cytochromes (Omc) such as OmcA and Mtr genes are also reported to be involved in the extracellular metal reduction (Fe(III)/Mn(Iv)/MFC electrode) pathway of Shewanella. ${ }^{15,17}$ In order to analyze the pilin and other outer membrane proteins involved in extracellular electron transfer and nanofilaments formation of the strain RP2, the genomic level comparison is required. ESI Table S1† shows the similarity percentage of Mtr gene sequences of Rhodopseudomonas palustris strain RP2 and other strains of Rhodopseudomonas palustris. ESI Fig. $\mathrm{S} 7 \dagger$ shows the phylogenetic relationship derived from amino acid sequence of MtrA gene alignments with closely related species. The two dimensional structural prediction of MtrA gene between Rhodopseudomonas palustris RP2 and Shewanella oneidensis MR1 is shown in ESI Fig. S8. $\dagger$ Alignment of MtrA protein of Shewanella oneidensis MR1 with Rhodopseudomonas palustris strain RP2 showed only 138 (18.0\%) identical sites. The major observation from our study is that no horizontal gene transfer events occurred at the genus level as can be seen in the phylogenetic relationships where the phylogenetic tree from these gene sequences shows the same relationships as seen for the general taxonomic classification of the same bacteria. Two dimensional structural predictions also show a higher degree of variation between these two strains.

Based on the above findings, we have demonstrated the physiological induction of nanofilaments from a metabolically versatile, iron(III) respiring, photosynthetic bacterium
Rhodopseudomonas palustris strain RP2. The nanofilaments produced by the strain RP2 are found to be conductive filaments which is evident from the ohmic behavior of their linear IV trace. Although the methods used in our study ${ }^{10,11,24,33}$ have provided conclusive evidence for the conductivity of nanofilaments from this Rhodopseudomonas palustris strain RP2, a complete understanding of the conductive nature of the biological proteins involved, molecular structure of filaments ${ }^{\mathbf{1 3 , 2 3 , 3 4}}$ and their electron exchange chain ${ }^{35,36}$ will deliver more information about the mechanism involved in conductivity. Further computational studies involving three dimensional homology modeling will reveal how structural changes in the outer membrane proteins of Rhodopseudomonas palustris strain RP2 influence its extracellular electron transfer mechanisms.

\section{Conclusions}

This study opens the possibilities of future research in the direction of bio-nanophysics ${ }^{\mathbf{1 3}}$ and genetic mutant studies ${ }^{\mathbf{1 2}}$ to get deeper understanding with the complete molecular composition and physiological process of induced nanofilaments. These findings expand our knowledge of the range of bacteria known to synthesize nanofilaments and provide further research opportunities in the field of bionanotechnology, bioelectrochemical remediation systems (petroleum hydrocarbon contaminated sites) and MMI process at photic environments.

\section{Experimental}

\subsection{Bacterial strain, growth conditions}

Rhodopseudomonas palustris strain RP2 was isolated from the anodic biofilm of a bioelectrochemical remediation system through serial dilution techniques. Cultures were routinely cultivated using anoxic rich medium (LB) in illuminated conditions. Nucleic acids were extracted and the 16S rRNA gene sequence of the strain was deposited in GenBank under the accession number (KJ460004). The cells were also examined for their growth under different physiological conditions such as photoorganotrophic, photolithotrophic, chemoorganotrophic and chemolithotrophic in anoxic and oxic environments. A chemically defined medium was used with acetate $(20 \mathrm{mM})$ and nitrate $(10 \mathrm{mM})$, sulfate $(10 \mathrm{mM})$, iron citrate $(10 \mathrm{mM})$ or iron(III) oxide $(10 \mathrm{mM})$ as the respective electron donor and acceptors supplemented with Wolfe's trace elements and vitamins as previously described. ${ }^{37}$

\subsection{Iron(III) oxide reduction}

For investigating the induction of extracellular filaments, cells were grown in two different environments: anoxic photoheterotrophic and chemoheterotrophic culture conditions supplemented with crystalline iron(III) oxide $(10 \mathrm{mM})$ as the terminal electron acceptor. The cells were grown in Wolfe's medium using acetate $(20 \mathrm{mM})$ supplemented with trace elements and vitamins. ${ }^{37}$ Iron(III) reduction was determined using the ferrozine assay. ${ }^{38}$ The bacterial suspension was added 
to a pre-weighed vial containing 0.5 M HCL. HCL extracted samples were added to $5 \mathrm{ml}$ of ferrozine $\left(1 \mathrm{~g} \mathrm{l}^{-1}\right)$ in $50 \mathrm{mM}$ HEPS buffer. The filtered samples were then analysed in a UV-Vis spectrophotometer (maxima@ $\lambda 562 \mathrm{~nm}$ ) to quantify the $\mathrm{Fe}$ (II) formation as previously described. ${ }^{38}$

\subsection{Microscopic methods}

4.3.1 Scanning electron microscopy. The bacteria and filament samples were collected from cultures grown under two different physiological conditions. Specialised sample preparation methods were employed in tandem with individual microscopic methods in order to preserve the nanowires along with the bacterial samples and to enhance image quality. The harvested cells and filaments were chemically fixed with $4 \%$ paraformaldehyde, $1.25 \%$ glutaraldehyde in PBS, $+4 \%$ sucrose, $\mathrm{pH}$ 7.2. The bacterial samples were filtered through the membrane filters with a pore size of $0.25 \mu \mathrm{m}$ using a sterile syringe, to remove the growth media from the cells. These filtered bacteria and filament samples were then washed gently several times in PBS buffer pH7 followed by deionised water. The samples were then dehydrated through a series of graded ethanol washes with increasing ethanol concentrations. Harvested bacterial samples were applied to graphite blocks. ${ }^{\mathbf{1 1}}$ The air-dried samples were observed under SEM (FEI Company, Model Quanta FEG 450) in ESEM mode without any coatings. ${ }^{24}$

4.3.2 Transmission microscopy. Filtered uranyl acetate was used as a negative staining agent. The harvested filament samples were placed on the TEM grids and then strained. The excess strain was removed by blotting with Whatman filter paper and the samples were then air dried before being observed under a TEM (JEM 1200 EXII, JEOL) at an accelerating voltage of $80 \mathrm{kV}$.

\subsubsection{Scanning probe microscopy}

4.3.3.1 Atomic force microscopy (ScanAsyst). Tapping mode AFM gives a higher resolution image of filament samples that are difficult to image through the current sensing mode. Samples were applied to freshly cleaved highly oriented pyrolytic graphite (HOPG) and left to adsorb for 30 minutes on the graphite surface. The adsorbed samples were fixed with $4 \%$ glutaraldehyde fixative for $10 \mathrm{~min}$. Samples were thoroughly washed with anaerobically prepared PBS buffer followed by subsequent anaerobic sterile deionised water to remove salts, and EPS (exo polysaccharides) from the cell surface. The HOPG samples with bacteria/nanofilaments were investigated using a ScanAsyst mode AFM (MultiMode 8 with Nanoscope V controller, Bruker, United States) as stated earlier. ${ }^{10,31}$ ScanAsyst is a self-optimising AFM imaging mode utilising an integrated Peak Force Tapping, which oscillates the cantilever below its resonance frequency. The premium ScanAsyst Air silicon nitride probes (nominal resonant frequency $50-90 \mathrm{kH}$, nominal spring constant $0.4 \mathrm{~N} \mathrm{~m}^{-1}$, nominal tip radius $10 \mathrm{~nm}$; Bruker, United States) were used. All AFM measurements were conducted inside a clean room (Class 1000) at $22^{\circ} \mathrm{C}\left( \pm 1{ }^{\circ} \mathrm{C}\right)$.

4.3.3.2 Current sensing AFM (CSAFM). Current sensing AFM (CSAFM) was used to image both topography as well as the conductivity of filament samples of the strain RP2 simultaneously. A bias voltage was applied to the sample while the cantilever was kept at virtual ground. A multipurpose scanner (Agilent multipurpose scanner, Australia) was employed with a CSAFM nose assembly for imaging the filaments as previously described. ${ }^{\mathbf{1 0 2 4}}$ The prepared AFM specimen was then mounted on a lab-built HOPG sample plates. The platinum coated probes were used with a nominal spring constant of $0.2 \mathrm{~N} \mathrm{~m}^{-1}$ for conductive imaging mode analysis. Topographic imaging of the bacterial nanofilaments was performed at a loading force of $5 \mathrm{nN}$. In addition to the topography, conductive mapping reveals information about the electrical properties of the filament and designated as nanofilaments of the strain RP2. ImageJ, WSxM, SPIP and Nanoscope software programs were used for further image data visualisation and processing of the SEM, SPM images.

4.3.3.3 Scanning tunnelling microscopy (STM). STM of air dried filament samples were investigated as stated earlier ${ }^{24,33}$ using a Bruker Multimode 8 STM equipped with 'E' scanner (maximum scan range $10 \mu \mathrm{m}$ in the in-plane $x$-and $y$-directions, and nominal $2.5 \mu \mathrm{m}$ in the normal to the surface $z$-direction) and Pt/Ir tips (Bruker, USA). Set point current values are in the range of 1-2 nA. The STM imaging was challenged by bacterial cells and organic contaminants. Samples were identified from flat features characteristic to HOPG surface. Image data processing was performed by using NanoScope analysis.

\subsection{Electrical transport measurements-nanofabricated electrodes}

To enable electrical measurements of nanofilament samples, combination of optical lithography, metal etching and dual beam focused ion beam (FIB) techniques were used as reported earlier. ${ }^{11,28}$

4.4.1 Defining the contact pads. Pyrex (Corning 7740) glass substrates were cleaned using Piranha solution for 30 minutes before being coated with chromium (Cr) and gold (Au). Electrode materials (10 $\mathrm{nm}$ of $\mathrm{Cr}$ as adhesion layer and $50 \mathrm{~nm}$ of $\mathrm{Au}$ as conducting layer) were deposited on the glass substrates using a sputter coater (TF500 from HHV) at a deposition rate of $25 \mathrm{~nm} \min ^{-1}$. Subsequently optical lithography was performed on the coated glass plates to define $400 \times 400 \mu \mathrm{m}^{2}$ contact pads with $50 \mu \mathrm{m}$ gap between the pads by spin coating them with a positive photoresist (AZ1518 photoresist from Clariant). Patterning was undertaken using a soda lime glass mask and EVG 620 Mask Aligner. The substrates were immersed in developing solution AZ 726 MIF with gentle agitation, selectively dissolving photoresists that were exposed to UV irradiation. The exposed metal layers were etched and the photoresist was removed as schematized in ESI Fig. S6. $\dagger$

4.4.2 Contacting the nanowires. Chemically fixed uncoated samples were placed on the glass plates with pre-patterned $\mathrm{Au}$ microelectrodes. The prepared samples were then rinsed and air-dried as previously reported. ${ }^{11}$ Before placing the samples in the dual beam focused ion beam system (FIB-SEM, FEI, Helios NanoLab), a $5 \mathrm{~nm}$ layer of Au was sputtered to avoid the charging effect of electron accumulation during the SEM imaging caused by the non-conductive glass plates. SEM 
imaging was used to identify the positions of nanowires located either between two adjacent contact pads or touching one contact pad. After locating a nanowire, ion beam deposition of Pt was then used to create contacting strips with $1 \mu \mathrm{m}$ wide and $1 \mu \mathrm{m}$ thickness using the in situ capability. In order to have a reference Pt strip, two adjacent contact pads were connected by a Pt strip bridge with the same geometry to quantify the resistance of the in situ Pt deposited strip per $\mu \mathrm{m}$ length.

4.4.3 I-V measurements. Current-voltage sweep measurements were performed at room temperature using an Agilent B1500A semiconductor parametric analyzer. Before proceeding with $I-V$ measurements a very short dip in $\mathrm{KI} / \mathrm{I}_{2}$ solution was used to remove the $\mathrm{Au}$ sputtered in order to avoid charging effect in the SEM column. Though the sputtered Au layer is very thin $(5 \mathrm{~nm})$, it is essential to fully remove prior to $I / V$ measurements. This removal was verified through various current-voltage measurements on adjacent and disconnected micro-electrodes that show infinity resistance. The sweeping voltage ranged from $-2 \mathrm{~V}$ to $+2 \mathrm{~V}$ in steps of $20 \mathrm{mV}$ and with a current compliance of $10 \mu \mathrm{A}$. This current compliance prevents damage (by joule heating) to the nanofilaments. The bacterial nanofilaments were tested in this way and their resistance was calculated from the ohmic trace of $I-V$. Resistivity was calculated from the length and resistance as follows

$$
R=\rho L / A
$$

where $R$ is measured resistance, $A$ is the cross sectional area of the nanofilament and $L$ is length of nanofilaments between two probes measured by FIB SEM. An open circuit control was conducted by depositing two Pt probes very close together on a prefabricated chip. The Pt reference strip showed zero current to the applied voltage at open circuit conditions which indicates the free of metal contamination space between the electrodes during the deposition.

\subsection{Genome sequencing and analysis}

Rhodopseudomonas palustris strain RP2 cells were grown in LB medium for $36 \mathrm{~h}$ and genomic DNA was isolated using the Mo Bio Ultraclean ${ }^{\circledR}$ Microbial DNA Isolation Kit. Genome sequencing was carried out in an ion torrent personal genome machine (PGM) platform using 314 chip as per the manufacturer's instructions at the Adelaide node of the Australian Genome Research Facility Ltd. (AGRF). Sequencing yielded 3255369 reads with a mean length of 173 bp resulting in $563.10 \mathrm{Mbp}$ sequenced bases and the longest read was $398 \mathrm{bp}$. De novo assembly and annotation of genome were carried out using CLC Genomics Workbench version 6.5 and rapid annotation using subsystem technology (RAST) version $4.0^{39}$ while phylogenetic analysis was conducted using MEGA version 5.2. ${ }^{\mathbf{4 0}}$

\section{Acknowledgements}

This work was performed at the South Australian node of the Australian National Fabrication Facility under the National Collaborative Research Infrastructure Strategy to provide nano and microfabrication facilities for Australia's researchers. The authors thank D. Venkatachalam, M. Krawsokoa, D. Simon for confirming the validity of the techniques used. KV thanks Australian Federal Government, University of South Australia for International Postgraduate Research Scholarship award (IPRS) and CRC CARE for the research top-up award.

\section{Notes and references}

1 D. R. Lovley, ASM News, 2002, 68(5), 231-237.

2 O. Bretschger, A. Obraztsova, C. A. Sturm, I. S. Chang, Y. A. Gorby, S. B. Reed, D. E. Culley, C. L. Reardon, S. Barua and M. F. Romine, Appl. Environ. Microbiol., 2007, 73(21), 7003-7012.

3 S. Kato, R. Nakamura, F. Kai, K. Watanabe and K. Hashimoto, Environ. Microbiol., 2010, 12(12), 3114-3123.

4 L. J. Liermann, E. M. Hausrath, A. D. Anbar and S. L. Brantley, J. Anal. At. Spectrom., 2007, 22(8), 867-877.

5 D. E. Holmes, K. T. Finneran, R. A. O'Neil and D. R. Lovley, Appl. Environ. Microbiol., 2002, 68(5), 2300-2306.

6 K. T. Finneran, R. T. Anderson, K. P. Nevin and D. R. Lovley, Soil and Sediment Contamination: An International Journal, 2002, 11(3), 339-357.

7 H. L. Ehrlich and D. K. Newman, Geomicrobiology, CRC press: Taylor \& Francis Group, 2008.

8 D. R. Lovley, D. E. Holmes and K. P. Nevin, Adv. Microb. Physiol., 2004, 49, 219-286.

9 R. A. Rosselló-Mora, W. Ludwig, P. Kämpfer, R. Amann and K. H. Schleifer, Appl. Microbiol., 1995, 18(2), 196-202.

10 G. Reguera, K. D. McCarthy, T. Mehta, J. S. Nicoll, M. T. Tuominen and D. R. Lovley, Nature, 2005, 1098-1101.

11 M. Y. El-Naggar, G. Wanger, K. M. Leung, T. D. Yuzvinsky, G. Southam, J. Yang, W. M. Lau, K. H. Nealson and Y. A. Gorby, Proc. Natl. Acad. Sci. U. S. A., 2010, 107(42), 18127-18131.

12 S. Pirbadian, S. E. Barchinger, K. M. Leung, H. S. Byun, Y. Jangir, R. A. Bouhenni, S. B. Reed, M. F. Romine, D. A. Saffarini and L. Shi, Proc. Natl. Acad. Sci. U. S. A., 2014, 111(35), 12883-12888.

13 D. R. Lovley and N. S. Malvankar, Environ. Microbiol., 2015, 17, 2209-2215.

14 J. K. Fredrickson and Y. A. Gorby, Curr. Opin. Biotechnol., 1996, 7(3), 287-294.

15 J. A. Gralnick and D. K. Newman, Mol. Microbiol., 2007, 65(1), 1-11.

16 D. J. Richardson, J. N. Butt, J. K. Fredrickson, J. M. Zachara, L. Shi, M. J. Edwards, G. White, N. Baiden, A. J. Gates and S. J. Marritt, Mol. Microbiol., 2012, 85(2), 201-212.

17 L. Shi, D. J. Richardson, Z. Wang, S. N. Kerisit, K. M. Rosso, J. M. Zachara and J. K. Fredrickson, Environ. Microbiol. Rep., 2009, 1(4), 220-227.

18 D. R. Lovley, J. D. Coates, E. L. Blunt-Harris, E. J. Phillips and J. C. Woodward, Nature, 1996, 382(6590), 445-448.

19 D. K. Newman and R. Kolter, Nature, 2000, 405(6782), 94-97. 20 S. E. Childers, S. Ciufo and D. R. Lovley, Nature, 2002, 416(6882), 767-769. 
21 C. Pfeffer, S. Larsen, J. Song, M. Dong, F. Besenbacher, R. L. Meyer, K. U. Kjeldsen, L. Schreiber, Y. A. Gorby and M. Y. El-Naggar, Nature, 2012, 491(7423), 218-221.

22 N. S. Malvankar, M. Vargas, K. P Nevin, A. E. Franks, C. Leang, B. C. Kim, K. Inoue, T. Mester, S. F. Covalla and J. P. Johnson, Nat. Nanotechnol., 2011, 6(9), 573-579.

23 N. S. Malvankar, M. Vargas, K. Nevin, P. L. Tremblay, K. Evans-Lutterodt, D. Nykypanchuk, E. Martz, M. T. Tuominen and D. R. Lovley, mBio, 2015, 6(2), e00084.

24 Y. A. Gorby, S. Yanina, J. S. McLean, K. M. Rosso, D. Moyles, A. Dohnalkova, T. J. Beveridge, I. S. Chang, B. H. Kim and K. S. Kim, Proc. Natl. Acad. Sci. U. S. A., 2006, 103(30), 11358-11363.

25 R. Whittenbury and A. McLee, Arch. Mikrobiol., 1967, 59(1-3), 324-334.

26 K. Eckersley and C. S. Dow, J. Gen. Microbiol., 1980, 119(2), 465-473.

27 F. W. Larimer, P. Chain, L. Hauser, J. Lamerdin, S. Malfatti, L. Do, M. L. Land, D. A. Pelletier, J. T. Beatty and A. S. Lang, Nat. Biotechnol., 2003, 22(1), 55-61.

28 G. Wanger, Y. Gorby, M. Y. El-Naggar, T. D. Yuzvinsky, C. Schaudinn, A. Gorur and P. P. Sedghizadeh, Oral Surgery, Oral Medicine, Oral Pathology and Oral Radiology, 2013, 115(1), 71-78.

29 L. A. Giannuzzi, B. Kempshall, S. Schwarz, J. Lomness, B. Prenitzer, and F. Stevie, Introduction to Focused Ion Beams, Springer, 2005, ch. 10.
30 S. M. Eichfeld, T. T. Ho, C. M. Eichfeld, A. Cranmer, S. E. Mohney, T. S. Mayer and J. M. Redwing, Nanotechnology, 2007, 18(31), 315201.

31 K. M. Leung, G. Wanger, M. El-Naggar, Y. Gorby, G. Southam, W. M. Lau and J. Yang, Nano Lett., 2013, 13(6), 2407-2411.

32 B. E. Logan, Nat. Rev. Microbiol., 2009, 7(5), 375-381.

33 J. P. Veazey, G. Reguera and S. H. Tessmer, Phys. Rev., 2011, E84(6), 060901-060904.

34 N. S. Malvankar and D. R. Lovley, ChemSusChem, 2012, 5(6), 1039-1046.

35 G. J. Bartlett, A. Choudhary, R. T. Raines and D. N. Woolfson, Nat. Chem. Biol., 2010, 6(8), 615-620.

36 M. J. Plevin, D. L. Bryce and J. Boisbouvier, Nat. Chem., 2010, 2(6), 466-471.

37 D. R. Lovley and E. J. Phillips, Geomicrobiol. J., 1988a, 6(3-4), 145-155.

38 D. R. Lovley and E. J. Phillips, Appl. Environ. Microbiol., 1988b, 54(6), 1472-1480.

39 R. K. Aziz, D. Bartels, A. A. Best, M. deJongh, T. Disz, R. A. Edwards, K. Formsma, S. Gerdes, E. M. Glass and M. Kubal, BMC Genomics, 2008, 9(1), 75.

40 K. Tamura, D. Peterson, N. Peterson, G. Stecher, M. Nei and S. Kumar, Mol. Biol. Evol., 2011, 28(10), 2731-2739. 